\title{
CAUCHY CONDITIONS ON SYMMETRICS
}

\author{
S. W. DAVIS ${ }^{1}$
}

\begin{abstract}
We call a symmetric $d$ on a space $X$ a wC symmetric if whenever $A \subseteq X$ and there exists $\varepsilon>0$ such that $d(x, y) \geqslant \varepsilon$ for all $x, y \in A$, then $A$ is relatively discrete. We show that there are no $L$-spaces which admit wC symmetrics. The wC notion is extended to certain weaker structures such as $\mathscr{F}$-spaces with similar results.
\end{abstract}

1. Introduction. A symmetric on a topological space $X$ is a distance function $d: X \times X \rightarrow[0, \infty)$ such that (i) $d(x, y)=0$ iff $x=y$, (ii) $d(x, y)=d(y, x)$ for all $x, y$, and (iii) $F \subseteq X$ is closed iff $d(x, F)>0$ for all $x \in X \backslash F$.

A space which admits a symmetric is called symmetrizable. A first countable symmetrizable $T_{2}$-space is a semimetrizable space, and the converse is true assuming all spaces are $T_{2}$, which we shall do.

Semimetrizable spaces and symmetrizable spaces have been studied for many years. Many of the properties of semimetrizable spaces have been shown to carry over to the non-first countable case, although in many cases the arguments have been much more difficult. There are a few notable exceptions, where properties do not carry over, see [B, S, DGN], and some questions still remain. In particular, we address in this note the following question:

Question 1.1 [Arhangel'skii, Rudin]. Is every regular Lindelöf symmetrizable space separible?

It will not be easy to find a counterexample, of course, since Lindelöf symmetrizable spaces are hereditarily Lindelöf $[\mathbf{N}]$. Thus a counterexample would be an $L$-space.

It may be that this question can be shown to have a positive answer, but at this writing that is not known. We provide a solution to the problem for a large class of symmetrizable spaces by considering a property related to Cauchy sequences.

\section{Cauchy conditions.}

Definition 2.1 [AN]. A symmetric $d$ on $X$ is called Cauchy iff $\lim _{n \rightarrow \infty} d\left(x_{n}, x_{n+1}\right)$ $=0$ whenever $\left\langle x_{n}: n \in \omega\right\rangle$ is a convergent sequence in $X$.

Subsequently, Arhangel'skii defined a weaker property which he called weakly Cauchy.

Definition $2.2\left[\mathbf{A}_{1}\right.$ ]. A symmetric $d$ on $X$ is called weakly Cauchy iff whenever $A \subseteq X$ and there exists $\varepsilon>0$ such that $d(x, y) \geqslant \varepsilon$ for all distinct $x, y \in A$, then $A$ is closed.

Received by the editors December 14, 1981 and, in revised form, March 9, 1982.

1980 Mathematics Subject Classification. Primary 54E20, 54D20, 54E25.

${ }^{1}$ This work was partially supported by a Miami University Summer Research Grant. 
Since symmetrizable spaces are sequential, it is clear that this is a weaker condition. The set $A$ in 2.2 could have no convergent sequences if the symmetric were Cauchy, and thus $A$ would be closed.

This condition was used by Nedev to partially answer 1.1 in [N].

THEOREM 2.3 [NEDEV]. A Lindelöf space which admits a weakly Cauchy symmetric is separable.

We now define an even weaker condition and show that 1.1 still has a positive answer in this case.

Definition 2.4. A symmetric $d$ on $X$ is called wC iff whenever $A \subseteq X$ and there exists $\varepsilon>0$ such that $d(x, y) \geqslant \varepsilon$ for all distinct $x, y \in A$, then $A$ is relatively discrete.

Since for any closed subset $A \subseteq X$, where $d$ is a symmetric on $X, d \mid A \times A$ is a symmetric for the relative topology on $A$, it is clear that any weakly Cauchy symmetric is a wC symmetric.

We now give an example of a wC symmetric which is not weakly Cauchy. This example has previously been described in [HS].

EXAmple 2.5. Let $X=\mathbf{R}$. Points of $\mathbf{R} \backslash\{0\}$ will have usual neighborhoods. A basic neighborhood of 0 will have the form $\{0\} \cup \cup\left\{\left(n-\varepsilon_{n}, n+\varepsilon_{n}\right): n \in T\right\}$ where $\varepsilon_{n}>0$ for each $n \in T \subseteq Z$ and $Z \backslash T$ is finite. (Here $Z$ denotes the integers.) Clearly $X$ is separable, hereditarily Lindelöf and not first countable since $\chi(0, X) \geqslant \omega_{1}$. We now define a symmetric $d$ on $X$ as follows:

$$
d(x, y)=d(y, x)=\left\{\begin{array}{cl}
0, & x=0=y, \\
|x-y|, & \text { if } x \neq 0 \text { and } y \neq 0, \\
1, & \text { if } x=0 \text { and } y \in \mathbf{R} \backslash Z \\
\frac{1}{|x+y|}, & \text { if } x=0 \text { and } y \in Z \backslash\{0\} \\
& \text { or } y=0 \text { and } x \in Z \backslash\{0\} .
\end{array}\right.
$$

It is easy to see that $d$ is a wC symmetric for $X$. However $Z \backslash\{0\}$, while relatively discrete, is not closed. Thus $d$ is not weakly Cauchy.

We note that any symmetric on a semimetrizable space is a wC symmetric. This is true since $x \in$ Int $B_{d}(x ; \varepsilon)$ for any $\varepsilon>0$ and $x \in X$ when $X$ is first countable and $d$ is a symmetric on $X$. Thus results obtained for wC symmetrics strengthen the corresponding results for semimetric spaces.

THEOREM 2.6. A Lindelöf space which admits a wC symmetric is separable.

PROOF. Since Lindelöf symmetrizable spaces are hereditarily Lindelöf, this will follow from the more general result 3.4 .

3. Weakly first countable spaces. The notion of $\mathrm{wC}$ symmetric can be easily extended to several weaker types of structures. 
Definition 3.1. A wfc-system for a space $X$ is a function $B: \omega \times X \rightarrow \mathscr{P}(X)$ such that for each $x \in X, B(n+1, x) \subseteq B(n, x)$ for each $n \in \omega$ and $\cap_{n \in \omega} B(n, x)=$ $\{x\}$, and $U \subseteq X$ is open iff for each $x \in U$ there exists $n_{x} \in \omega$ with $B\left(n_{x}, x\right) \subseteq U$.

A space which admits a wfc-system is called weakly first countable $\left[\mathbf{A}_{2}\right]$.

Definition 3.2. An F-system for a space $X$ is a wfc-system for $X$ with the additional property that if $F \subseteq X$ is closed and $x \notin F$ then there exists $n \in \omega$ such that for any $y \in B(n, x) \backslash\{x\}$ there exists $n_{y} \in \omega$ such that $\{x, y\} \nsubseteq$ $\cup_{z \in F} B\left(n_{y}, z\right)$.

A space which admits an $\mathscr{F}$-system is called an $\mathscr{F}$-space [HS].

Definition 3.3. A wfc-system $B$ for $X$ is called wC iff whenever $A \subseteq X$ and there exists $n \in \omega$ such that $B(n, x) \cap A=\{x\}$ for each $x \in A$, then $A$ is relatively discrete.

If $d$ is a symmetric on $X, B(0, x)=X$, and $B(n, x)=\{y: d(x, y)<1 / n\}$ for $n \in \omega \backslash\{0\}$, then $B$ is a wfc-system (in fact, an $\mathscr{F}$-system) for $X$, and $B$ is wC iff $d$ is wC.

THEOREM 3.4. A hereditarily Lindelöf space which admits a wC F-system is separable.

Proof. Suppose $X$ is hereditarily Lindelöf and $B$ is a wC F $F$-system on $X$. Let $D_{0}$ be the set of isolated points in $X$. Since $X$ is hereditarily Lindelöf, $D_{0}$ is countable. For $n \in \omega \backslash\{0\}$, we define $D_{n}$ as follows: $D_{n}$ is a maximal subset of $X$ such that $x \in D_{n}$ implies $B(n, x) \cap D_{n}=\{x\}$. Since $B$ is wC, $D_{n}$ is countable for each $n>1$.

We now show $D=\cup_{n \in \omega} D_{n}$ is dense. Suppose $U \neq \varnothing$ and $U$ is open. If $U \cap D=\varnothing$, then we choose $x \in U$, and since $B$ is an $\mathcal{F}$-system there exists $n \in \omega$. such that for $y \in B(n, x) \backslash\{x\}$ there exists $n_{y} \in \omega$ such that $\{x, y\} \nsubseteq$ $\cup_{z \in D} B\left(n_{y}, z\right)$. Since $x$ is not isolated, choose $y \in B(n, x) \backslash\{x\}$, and $k \geqslant n_{y}$ such that $B(k, x) \subseteq U$ and $B(k, y) \subseteq U$. Now either $D_{k} \cup\{x\}$ or $D_{k} \cup\{y\}$ will contradict the maximality of $D_{k}$, and the proof is complete.

We turn now to the question of when a weakly first countable space has a wC wfc-system. It is clear that if $B$ is a hereditary wfc-system, then $B$ is wC. It follows from known results that this is quite a restriction.

TheOREM 3.5. If $B$ is a hereditary wfc-system for $X$, then $\{B(n, x): n \in \omega\}$ is $a$ neighborhood base at $x$.

Proof. Obviously, it is enough to show that for each $n \in \omega, x \in \operatorname{Int} B(n, x)$. Since weakly first countable spaces are sequential [HS], $X$ is hereditarily sequential. Thus $X$ is Frechét $\left[\mathbf{A}_{3}\right]$, and hence $x \in \operatorname{Int} B(n, x)$ for each $n \in \omega[\mathbf{H S}]$.

Burke has shown in $[\mathrm{Bu}]$ that every semimetrizable space has a compatible weakly Cauchy symmetric. It is natural to ask if a similar theorem can be obtained for symmetrizable spaces. The space in Example 2.5 has a compatible weakly Cauchy symmetric. For regular spaces this remains open; however, Kofner has given in [K], a $T_{2}$ symmetrizable space which does not even admit a wC wfc-system.

\section{Questions.}

Question 4.1. Does every symmetrizable $T_{3}$ space have a compatible wC symmetric, or even a compatible wC $\mathscr{F}$-system? 
An affirmative answer to 4.1 would show that there are no symmetrizable $L$-spaces, answering question 1.1 .

Question 4.2. Does every space with a compatible wC symmetric have a compatible weakly Cauchy symmetric?

As noted in §3, the answer is "yes" for first countable spaces and also for Example 2.5.

\section{REFERENCES}

[A] P. S. Alexandroff and V. V. Nemytskiu, Metrizability conditions for topological spaces and the axiom of symmetry, Mat. Sb. 3 (1938), 663-672.

[A $]$ A. V. Arhangel'skĩ, Behavior of metrizability under factor mappings, Soviet Math. Dokl. 6 (1965), 1187-1190.

$\left[\mathbf{A}_{2}\right]$ _ Mappings and spaces, Russian Math. Surveys 21 (1966), 115-162.

$\left[\mathrm{A}_{3}\right] \ldots$, A characterization of very $k$-spaces, Czechoslovak Math. J. 18 (1968), 392-395.

[B] D. A. Bonnett, A symmetrizable space that is not perfect, Proc. Amer. Math. Soc. 34 (1972), 560-564.

[Bu] D. K. Burke, Cauchy sequences in semimetric spaces, Proc. Amer. Math. Soc. 33 (1972), 161-164.

[DGN] S. W. Davis, G. Gruenhage and P. J. Nyikos, $G_{\delta}$-sets in symmetrizable and related spaces, Topology Appl. 9 (1978), 253-261.

[HS] P. W. Harley and R. M. Stephenson, Symmetrizable and related spaces, Trans. Amer. Math. Soc. 219 (1976), 89-111.

[K] J. A. Kofner, On a new class of spaces and some problems of symmetrizability theory, Soviet Math. Dokl. 10 (1969), 845-848.

[N] S. Nedev, Symmetrizable spaces and final compactness, Soviet Math. Dokl. 8 (1967), 890-892.

[S] R. M. Stephenson, Near compactness and separability of symmetrizable spaces, Proc. Amer. Math. Soc. 68 (1978), 108-110.

DePartment OF MATHEMATICS, Miami University, OXFORD, OHIO 45056 